\title{
Le père insuffisant. Remarques sur la phraséologie politique en Chine ancienne
}

The Insufficient Father. Reflections on Early Chinese Political Rhetoric

不够充分的父親一一古代中國政治用語發微

Jean Levi

\section{OpenEdition}

\section{Journals}

Édition électronique

URL : http://journals.openedition.org/extremeorient/208

DOI : 10.4000/extremeorient.208

ISSN : 2108-7105

\section{Éditeur}

Presses universitaires de Vincennes

Édition imprimée

Date de publication : 1 janvier 2012

Pagination : $51-82$

ISSN : 0754-5010

Référence électronique

Jean Levi, «Le père insuffisant. Remarques sur la phraséologie politique en Chine ancienne », ExtrêmeOrient Extrême-Occident [En ligne], Hors-série | 2012, mis en ligne le 01 janvier 2015, consulté le 30 avril 2019. URL : http://journals.openedition.org/extremeorient/208 ; DOI : 10.4000/extremeorient.208 


\title{
Le père insuffisant. \\ Remarques sur la phraséologie politique en Chine ancienne
}

\author{
Jean Levi
}

\section{Les temps idylliques des Shang et des premiers Zhou : le roi père réel de son peuple}

Dans une étude désormais classique sur les institutions de la Chine archaïque, Léon Vandermeersch faisait remarquer que la royauté Shang dérivait selon toute vraisemblance du pouvoir paternel. Tout au moins c'est ce que semble attester le caractère wang 王 «roi ». Celui-ci se confond à l'origine avec le caractère $s h i \pm$ dont l'éminent épigraphiste Guo Moruo a pu démontrer qu'il avait originairement le sens de «mâle». Mais contrairement à ce qu'a pu soutenir Guo Moruo, la représentation pictographique du terme désignant la royauté par un sexe en érection renvoie non pas à un culte phallique propre à toute religion primitive, mais à la paternité dont la virilité est l'expression. «Le roi wang est le "viril" $s h i^{1}$ » explique Léon Vandermeersch, parce qu'il est le père de l'ethnie, héritier de la puissance de l'ancêtre fondateur. À ce titre, il est le seul vrai père de la communauté. Les autres pères, dépossédés de leur puissance paternelle, ne forment plus qu'une classe d'âge et sont ravalés au rang de simples «pèresoncles », les fu. Non que les oncles soient devenus des pères, mais le père ayant été privé de sa qualité d'ascendant dans le culte ancestral n'est plus qu'un oncle. En effet, dans le système religieux de la dynastie Shang, seul le Chef était en droit d'offrir des sacrifices aux ancêtres et aux entités tutélaires de la communauté, et tirait son autorité de cette prérogative; si bien que tout se passait, grâce au culte sacrificiel, comme si l'ethnie tout entière était constituée des enfants, $z i$, d'un seul père, le roi. Les relations de filiations réelles se sont ainsi trouvées supplantées par cette relation symbolique; une relation symbolique qui, pour

1. Par la suite, le terme en est venu à désigner, en chinois classique, la classe des gentilshommes. Voir Vandermeersch (1977-1980): II, 18. 
être de l'ordre de l'imaginaire, n'en déterminait pas moins les formes réelles de l'organisation politique. La communauté se subdivisait en classes d'âge formées des «pères» $(f u)$, des «mères» $(m u)$, des «aïeux» $(z u)$ et des «aïeules» $(b i)$. Les distinctions entre groupes familiaux ou claniques se trouvaient dès lors effacées; la division du corps social se faisait soit par essaimage du groupe en «maisons territoriales» extra-métropolitaines qui se trouvaient reliées au centre dirigeant par le culte ancestral dont le roi était l'unique officiant, en sorte qu'elles ne jouissaient d'aucune autonomie religieuse, soit à la métropole, par spécialisation en corps de métiers, certains groupes en venant à être affectés à des tâches spécifiques. Mais toutes ces divisions s'inscrivaient dans le cadre des relations de parenté: les caciques yin, ainsi que l'atteste la similitude des graphies du mot les désignant et du caractère $f u$, étaient issus de la collectivité des pères-oncles dans leur rôle de conseillers du roi; de même le mot $z i$, les fils, en vint à ne plus désigner que les membres de la communauté Yin les plus proches du chef et non pas l'ethnie dans son entier, tandis qu'apparaissait la catégorie des chen, les simples «sujets». Puis, le processus de différenciation sociale continuant à œuvrer, de nouvelles divisions apparurent et le mot chen se spécialisa à son tour pour désigner la fonction et le rang de «féal». Dans cette société, structurée selon l'axe vertical de subordination au Père et l'axe latéral de proximité ou d'éloignement par rapport au Chef, les trois ordres religieux, familial et politique coïncident pour embrasser l'ensemble de la communauté des Shang. La royauté chinoise ancienne présente donc le trait remarquable d'être un patriarcat «institué non par superposition du pouvoir supérieur d'un patriarche aux pouvoirs respectifs de tous les pères de leur position paternelle, mais par éviction de tous les pères de leur position paternelle réservée au seul roi $^{2} \gg$. En d'autres termes, selon Léon Vandermeersch, le roi n'est un sur-père que parce que tous les autres pères sont des sous-pères; son autorité s'établit donc moins sur une addition que sur une soustraction des pouvoirs paternels. La dynastie des Zhou, qui succéda à celle des Shang-Yin au XI ${ }^{e}$ siècle avant notre ère, tout en procédant à des aménagements considérables de l'organisation étatique de ses devanciers, se conforma au même schéma. Le roi reste un père et les structures politiques demeurent adossées à la parenté; toutefois ces relations sont «réorganisées de manière à composer au-dessous de la souveraineté royale un ensemble savamment articulé de souverainetés dérivées ${ }^{3} »$. C'est ainsi que se constitue le système classique du culte aux ancêtres. La société noble se trouve enserrée dans le double réseau du culte majeur et du culte mineur, instaurant un pouvoir paternel par filiation, et une structuration hiérarchique par bifurcation

2. Vandermeersch (1977-1980): II, 18.

3. Billeter (1991): 881 . 
collatérale, assurant sa diffraction dans l'ensemble de la communauté noble. De la sorte, tout comme sous les Shang, mais de façon plus souple, le pouvoir politique ne fait que doubler les relations hiérarchiques de la parenté réelle, auxquelles le culte rendu aux ancêtres confère une dimension religieuse. Le souverain, de par sa position au sein de la maison régnante, est le «père» de la communauté cultuelle et culturelle formant l'empire Zhou en tant que descendant de l'ancêtre fondateur de la race, tandis que les chefs des seigneuries, qui sont pour la plupart les descendants de cadets de la lignée principale, sont des pères pour les sujets des principautés sur lesquelles ils règnent en tant que chefs du culte seigneurial et fédèrent sous leurs ordres tous les «pères »- les grands officiers, chefs des cultes mineurs, descendants de cadets fondateurs d'une lignée par bifurcation à partir de la lignée collatérale d'un cadet de la branche principale des Zhou. Les responsabilités exercées par les membres de la noblesse étaient déterminées par leur place dans la hiérarchie des cultes aux ancêtres. Plus le nombre d'ancêtres individués auxquels le noble est en droit de sacrifier est important, plus il jouira d'un rang élevé. Inversement, tout droit à un sacrifice signifie qu'on occupe une place dans une hiérarchie nobiliaire et qu'on remplit nominalement, sinon en fait, une fonction héréditaire ${ }^{4}$.

Cependant, dans le nouveau système, le souverain est un sur-père en tant qu'il est le patriarche au-dessus de tous les pères. Ainsi trouve-t-on dans le paragraphe XXI du Laozi cette curieuse formule:

De jadis à aujourd'hui, le Nom seul jamais ne faillit, permettant grâce à lui de remonter aux patriarches des êtres ${ }^{5}$.

L'expression chinoise rendue par «patriarche des êtres» est zhongfu, mot à mot: «les pères de la masse», binôme qui désigne les seigneurs chefs de lignée. Dans un dialogue du Zhuangzi, un anachorète du nom de Xuyou dissuade le sage Yao de céder son trône à son propre maître, en lui faisant valoir qu' «il y a l'ancêtre clanique des groupes lignagers (zhongfu fu) et l'ancêtre lignager (zhongfu); son maître pourrait être un père de la foule [des membres du groupe lignager] (zhon$g f u)$, mais non le père des pères de l'ensemble des membres du groupe lignager

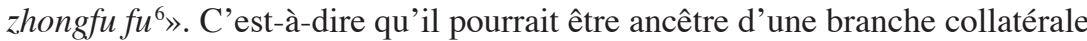
mais non pas l'ancêtre primordial auquel remontent tous les différents ancêtres

4. Le Livres des rites (Liji), t. 2, «Jifa» («Règles sacrificielles»), Couvreur (1950): 264-265.

5. Le manuscrit de Mawangdui porte, au lieu de $f u$ 甫, désignation honorifique de l'homme après la prise du bonnet viril, et par extension «éminent», « début», l'homonyme $f u$ 父 signifiant «père » et par extension «ancêtre», dont le caractère $f u$ 甫 «éminent», peut être par ailleurs un substitut phonique.

6. Zhuangzi, XII, trad. Levi (2006): 97. Le Heguanzi au chapitre IX utilise la même expression de zhongfu «des pères de lignées » comme périphrase désignant les seigneurs 


\section{Jean Levi}

des lignées. En d'autres termes, les relations hiérarchiques de parenté pouvant se transcrire sur le plan de la souveraineté, il pourrait être seigneur mais non pas empereur. Dans le Laozi, la formule renvoie donc, en recourant aux catégories de la religion constituée du culte aux ancêtres, aux entités transcendantes dont le divers phénoménal est issu. L'expression zhongfu - et plus encore celle de zhongfu fu - confère ainsi à la souveraineté sa forme la plus absolue en en dévoilant les soubassements claniques.

\section{Le souverain comme fils du Ciel et Ciel du peuple}

Si le roi des Zhou est un père - le père du peuple Zhou -, il est aussi un fils; car tout père se positionnant sur l'axe vertical des filiations est pris dans une double relation: celle vis-à-vis de sa descendance et celle vis-à-vis de ses ascendants; or le Ciel étant le géniteur de tous les êtres, le Chef a pour premier ancêtre le Ciel; il est donc en quelque manière «Fils du Ciel», et c'est en tant que père de la race qu'il est habilité à rendre un culte suprême au Ciel, ce qui en fait un fils, de même que, réciproquement, il est père du peuple Zhou dans la mesure où, Fils du Ciel, il rend un culte à celui-ci et se signale comme Chef suprême de l'ensemble des collèges cultuels dérivant du tronc principal ${ }^{7}$. Mais en fait, ce n'est pas simplement parce que le Ciel est l'ancêtre de tous les êtres que le maître de la communauté des hommes en est le fils, mais aussi parce que le Ciel est réellement le grand ancêtre de la maison royale des Zhou. Certains hymnes, proférés au moment du grand sacrifice au Ciel et relatant la geste de l'ancêtre fondateur des Zhou, Houji, le Souverain Millet, laissent entendre que le sacrifice au Ciel fut institué par leur illustre aïeul ${ }^{8}$.

qui se trouvent aussi être chefs des collèges du culte aux ancêtres en tant qu'aînés issus de la branche aînée d'un groupe clanique. Levi (2008): 64.

7. La section «Jiao tesheng» («Une victime unique pour le sacrifice dans la banlieue») du Liji fait remarquer que, de même que tous les êtres tirent leur origine du Ciel, de même l'homme tire son origine de ses ancêtres. Voilà pourquoi on associait le Souverain Millet à Shangdi, le dieu suprême. Par le sacrifice dans la banlieue, le Souverain «entendait rendre grâce à l'origine de toute chose et faisait retour au commencement de sa lignée». Voir Couvreur (1950): t. 1, IX, 594.

8. «Depuis que le Souverain Millet (Houji) a institué le sacrifice, jamais il n’y eut ni faute ni négligence, depuis ce jour jusqu'à aujourd'hui » dit l'hymne «Shengmin » du Daya qui retrace la carrière quasi divine de l'ancêtre des Zhou. Sa mère, Jiang Yuan, désespérant de rester sans enfant, invoqua la divinité par un grand sacrifice yin; le dieu agréa sa prière et laissa sur terre une empreinte de pied gigantesque. Jiang Yuan, ayant marché sur la marque de son orteil, devint grosse et mit au monde un fils aux pouvoirs miraculeux. Pour avoir apporté aux hommes le don des grains, il reçut le nom de Souverain Millet; par ailleurs, grâce à l'abondance des récoltes il put instaurer un culte au seigneur du Ciel. 
Le récit veut légitimer le pouvoir des Zhou en retraçant leur filiation divine: par le Souverain Millet, ils se rattachent à l'Empereur d'en-Haut, ils sont véritablement «Fils du Ciel» et habilités à lui rendre un culte, comme des descendants rendent un culte à leurs aïeux.

Zhuangzi, dans un dialogue mettant aux prises Confucius et son disciple favori Yan Hui, a sans doute en tête cette élaboration conceptuelle quand il fait dire à ce dernier: "Alors ferme sur le fond, je me montrerai souple dans les apparences. J'userai de formules toutes faites, et me cacherai derrière les précédents. Étant ferme sur l'essentiel, je me place sous la tutelle du Ciel; me sachant autant que le Fils du Ciel son fils, je parlerai sans me soucier qu'il m'approuve ou me désapprouve. Se comporter ainsi c'est posséder la candeur des enfants. Voilà ce que j'appelle se mettre sous la tutelle du $\mathrm{Ciel}^{9}$.»

La métaphore paternelle vaut dès lors dans les deux sens, car si le Ciel est un père pour le souverain, celui-ci est un Ciel pour le peuple. De nombreux textes, assez tardifs il est vrai, mais qui jouent sur des représentations anciennes, assimilent Ciel et souverain. Ainsi dans un chapitre au titre symptomatique de «L'exemple du Ciel», le Heguanzi a ce développement visant à fusionner les deux entités l'une dans l'autre par la simple élision du sujet en sorte que l'on ne sait plus où commence l'action du souverain et où finit celle du Ciel:

Telle est la façon dont le Ciel contrôle le manche du boisseau. Depuis le centre, il assigne à chacun sa place après examen; il gouverne au moyen des quatre influx : l'étoile de l'Extension devant, l'étoile polaire derrière, l'étoile de la corne du Bélier à gauche, et la Hallebarde à droite. Il scrute les configurations et se conforme à la raison des choses; il inspecte les fonctionnaires et la foule du peuple, de telle sorte que chacun, petit ou grand, remplit son office. Ne suscitant jamais ni rancœur ni récriminations, ayant une conduite irréprochable et une réputation sans tache, il fait rayonner son prestige jusqu'aux confins de l'univers et étend son autorité sur tous les êtres de la création, tandis que ses bienfaits répandent leur rosée sur les quatre orients sans rencontrer d'obstacles ${ }^{10}$.

Ou encore dans un autre chapitre, comme pour mieux enfoncer le clou:

Dans un autre hymne sacrificiel, le poème semble sous-entendre que c'est sur ordre de l'empereur d'en-Haut que le Souverain Millet apporta la culture céréalière aux hommes : «Je pense à toi, Houji, prince civilisateur. Tu es associé au Ciel. Tu nous as apporté les grains, il n'est plus haut bienfait; tu nous as fait don du blé et de l'orge, sur ordre de l'empereur du Ciel qui nous les destinait pour notre nourriture. Sans distinction de contrée ni de frontière, tu as fait observer les lois. (Shijing, «Siwen», trad. Couvreur (1966): 420.)

9. Zhuangzi, IV, trad. Levi (2006): 36 .

10. Ho-Kouan-Tseu, I, trad. Levi (2008): 26. 


\section{Jean Levi}

Telle est la racine des dix mille êtres, les portes et les fenêtres du ciel et de la terre, la plénitude du Tao et de son efficace... Le Prince est comme le Ciel. Si le Ciel n'ouvre pas ses fenêtres, les subordonnés peuvent en subir du dommage. L'Homme unique, racine de toute chose, peut être appelé $\mathrm{Ciel}^{11}$.

Lui répond en écho cette définition de la souveraineté impériale fournie par le Wenzi: «On appelle Fils du Ciel celui qui, détenteur de la Voie propre au Ciel, est en mesure de gouverner l'empire ${ }^{12}$.» Le Ciel dispense sa manne sur le peuple et veille à leur entretien: «Le Ciel couvre tous les êtres, leur prodiguant ses dons et pourvoyant à leur entretien; il donne sans jamais prendre ${ }^{13} »$, dira le même ouvrage; de même, le souverain dispense ses largesses au peuple et lui assure confort et sécurité, il se signale lui aussi par cette capacité à donner inhérente à l'action céleste: «Dans les temps anciens, les souverains [...] manifestaient leur pouvoir en prêtant vie et non en infligeant la mort, en donnant et non en prenant ${ }^{14}$.» À l'instar du Ciel qui couvre tous les êtres et pourvoit, avec la collaboration de la Terre, à tous leurs besoins, le rôle du souverain est de prodiguer ses dons à tous les êtres de la création. Et ce, sans même avoir besoin de recourir à la parole, étant donné que, ainsi que Confucius l'a énoncé avec force, «le Ciel ne parle pas»:

Lorsque le Saint est au pouvoir, il se contente d'abriter le Tao en son sein, pour que ses bienfaits se répandent sur tous les peuples de l'univers sans qu'il lui soit besoin de s'exprimer. Ah, qu'il est profus, qu'il est grand, l'enseignement sans parole ${ }^{15}$ !

Mais l'assimilation du souverain au Ciel ne se cantonne pas à la phraséologie politico-cosmique des traités de gouvernement des Royaumes combattants et des Han. Elle est inscrite dans la pratique rituelle effective, à travers les deux institutions cardinales de la royauté Zhou qui se perpétueront bien après la chute des Zhou, sous la dynastie Han et même jusqu'au $\mathrm{Xx}^{\mathrm{e}}$ siècle pour l'une d'entre elles tout au moins. Il s'agit de la déambulation royale dans le Mingtang, le «Palais des Lumières », et du jiao, le grand sacrifice au Ciel effectué par l'empereur dans la banlieue sud. Il ne saurait être question de fournir, dans le cadre de cette étude, une analyse exhaustive de ces deux rituels fondamentaux. Disons simplement ici que par ces deux actes cérémoniels, le souverain s'appariait au Ciel tout en convertissant le mouvement céleste en norme humaine et sociale par la seule vertu du cérémonial.

11. Ho-Kouan-Tseu, VI, trad. Levi (2008) : 39-40.

12. Wenzi, trad. Levi (2008): VIII, 12.

13. Wenzi, trad. Levi (2008): VII, 5c.

14. Wenzi, trad. Levi (2008): XII, 2.

15. Huainanzi (2003): VI, 4a. 
Le Mingtang est un bâtiment qui se donne pour la réplique du cosmos avec son toit rond comme le Ciel et sa base carrée comme la Terre comportant quatre façades orientées selon les points cardinaux autour d'une salle centrale. Chacun des espaces latéraux était divisé en trois pièces. Ainsi figurait-il la totalité de l'univers spatio-temporel. Le Fils du Ciel s'y déplaçait afin de correspondre à la saison convenable et de signifier, de par sa position, la configuration générale du moment. Il y proclamait, dans la chambre adéquate, revêtu d'une robe aux couleurs de la saison, la forme du gouvernement, la musique, les saveurs, les viandes, les sacrifices et les rites appropriés. Par sa marche conforme au cours du calendrier, dont elle assurait en retour la stabilité, il manifestait l'intime solidarité qui le reliait au Ciel. De la même façon, le grand rite solennel de la banlieue sud où le souverain, en qualité de Fils du Ciel, sacrifiait un jeune taureau roux, tout en y associant l'ancêtre primordial de la maison régnante, Houji, le Souverain Millet, portait témoignage des liens privilégiés de l'empereur avec le Ciel. Mais encore il visait à souligner le parallélisme de leur action bienfaisante, grâce à l'association du culte aux ancêtres du sacrifice rendu au Ciel. Le rite se clôt en effet par une distribution générale de nourriture, en sorte que le sacrifice au Ciel est le modèle aussi bien de l'ordre cosmique que du fonctionnement de la société. À la fin du sacrifice à Houji qui suit celui au Ciel, où la victime est entièrement consumée par les flammes, les parts sacrificielles sont réparties en cercles de plus en plus larges jusqu'à leur complet épuisement ${ }^{16}$. Si les restes disparaissent en étant consommés, ils laissent néanmoins une trace qui est la reconnaissance d'une dette et l'instauration d'un devoir liant le subordonné à son supérieur en retour du reste reçu, le bao. C'est là une notion fondamentale, car elle commande la piété filiale et les qualifications hiérarchiques qu'instaure la loi du devoir. Derrière la métaphore céleste se cache encore la figure du Père et ce, de multiples façons. Tout d'abord, sur le plan cosmogonique, en tant qu'ancêtre primordial ayant donné naissance à tous les êtres et assurant leur subsistance, il est un père pour les êtres vivants, rôle qui se manifeste précisément dans l'action de grâce sacrificielle, obligation symétrique à la piété filiale, en ce que l'une comme l'autre valent comme don en retour. Les fils et les peuples ont ceci de commun qu'ils sont en dette: les uns réellement vis-à-vis de leurs

16. Liji, «Jitong», voir Couvreur (1950) (t. 2, vol. I, 328) : «Tous les bienfaits reçus en grand nombre du Ciel devaient se répercuter jusqu'en bas de la société. La seule différence entre eux étaient que les uns en bénéficiaient avant les autres. Il ne pouvait être question que les supérieurs accaparent pour eux les biens et laissent le peuple mourir de faim ou de froid. Quand les grands recevaient de grands bienfaits, les inférieurs s'attendaient à les voir s'écouler vers le bas; ils savaient qu'ils recevraient nécessairement quelque chose. Et ils le savaient du spectacle offert par la manducation des viandes sacrificielles. C'est pourquoi les sages ont dit que le sacrifice fournissait le modèle du gouvernement.» 


\section{Jean Levi}

géniteurs qui les ont engendrés et nourris, les autres vis-à-vis du Fils du Ciel, lequel assure fictivement la distribution de la manne céleste à tous ses sujets. En second lieu, sur le plan numérologique, sous les espèces du trigramme Qian, symbole du masculin, il forme une paire avec le trigramme Kun, la Terre, paire qui renvoie aux deux rubriques maîtresses yin et yang entre lesquelles s'ordonne l'ensemble des existants. Il est donc l'emblème de la paternité dans ce qu'elle a de plus éminent.

\section{Le souverain comme «père du peuple»}

Cette homothétie des deux ordres familial et politique se manifeste de façon exemplaire dans la piété filiale. Mais celle-ci n'est que la traduction dans le code des devoirs sociaux de l'identification de la figure du souverain à celle du père. L'adéquation entre les deux rôles trouve son expression la plus accomplie dans les obligations luctuaires qui imposent le même temps de deuil et les mêmes manifestations de chagrin pour un souverain que pour un père. L'assimilation du père au souverain est un poncif de la littérature politique des Royaumes combattants. L'un appelle immanquablement l'autre. Comme si on ne pouvait parler de monarque sans que se dessine en surimpression l'image paternelle. Le balancement rhétorique qui associe les deux figures revient dans la prose chinoise avec une fréquence lancinante. L'identité souverain/père fait partie de ces évidences jamais remises en question, car elles font partie de l'impensé de la pensée: «Qui veut observer un pays en observe le prince, qui veut observer une famille en observe le père. Car qui sait gouverner un pays se comporte en prince, qui sait diriger une famille se comporte en père ${ }^{17} »$, enjoint un paragraphe de la première section des Quatre canons de l'empereur Jaune, qui date probablement du début du $\mathrm{III}^{\mathrm{e}}$ siècle av. J.-C. L'association des deux symboles de l'autorité fait partie de cette armature intangible des dictons qui préside à la sagesse des nations. Dans le Han Feizi, Guan Zhong, interrogé sur son lit de mort par son prince, le duc Huan de Qi, sur les collaborateurs capables de lui succéder, répond par un proverbe qui les met en parallèle: «On dit d'ailleurs que nul n'est mieux placé qu'un roi pour connaître ses sujets; de même que personne ne connaît mieux ses fils qu'un père ${ }^{18}$. » Dans la Dispute sur le sel et

17. Huangdi sijing, «Les six alternatives», trad. Levi (2009): 136.

18. Han Feizi X, «Les dix erreurs», trad. Levi (1999): 127. Le parallélisme est omniprésent chez Han Fei: «On m'a inculqué cette règle: le sujet sert son roi, le fils son père, la femme son mari; ces trois soumissions respectées, le monde est en paix; bafouées, il est troublé. Telle est la règle immuable qui préside à l'empire.» (chap. LI: 538-39); ou bien: «Je pense, quant à moi, qu'il est du devoir de tout être humain de servir son prince et d'entretenir ses parents. Qui sert son prince et entretient ses parents ne saurait 
le fer, où les deux parties en présence, les lettrés confucéens et les grands commis de l'État légistes, se renvoient la balle des vérités premières, il est fait des deux côtés un large usage de l'analogie à des fins démonstratives: «À la mort d'un prince, les ministres ne changent pas sa politique, à la mort d'un père, les fils ne modifient pas ses orientations», dira le grand secrétaire pour justifier la poursuite de la politique belliciste de Han Wudi ${ }^{19}$. «Un père n'exige pas trop de ses enfants; un prince ne demande pas l'impossible à ses sujets ${ }^{20} »$, argueront les lettrés pour inciter le camp adverse à plus de clémence. Tandis que les ministres ont justifié un peu plus tôt leur ligne répressive par cette comparaison quelque peu éculée: «L'État est à l'égard du peuple comme un père compatissant avec ses fils. Peut-on être loyal sans sermonner, et aimant sans beaucoup exiger?»

Zhuangzi, dénonçant l'inconséquence de l'humaine nature, les associe lui aussi, comme par-devers lui, comme s'il y était contraint par la force d'attraction qu'exercent l'un sur l'autre le fils pieux et le loyal sujet: «On considère qu'il est du devoir d'un fils de ne pas flatter ses parents et d'un sujet de ne pas flagorner son prince, que ce sont là les marques les plus hautes de la piété et de la loyautéên.»

Parfois, par la vertu du strict parallélisme des sentences commandé par les lois de la stylistique, les deux figures se confondent à tel point que le père fait office de prince (et réciproquement): «Il est du devoir du sujet d'être prêt à se dévouer pour son prince dans l'adversité en payant de sa personne; comme il est dans les attributions d'un père compatissant de veiller à nourrir ses enfants quand ils ont faim, et à les vêtir quand ils ont froid ${ }^{22}$ », dira le Wenzi, condensant un développement un peu verbeux du Huainanzi.

Lu Jia, que le fondateur des Han avait chargé de rédiger un traité de gouvernement pouvant servir de programme politique à la nouvelle dynastie, inscrit cette double relation dans le marbre de la cosmogonie: «Le premier saint leva son regard vers le Ciel pour observer les signes célestes, il l'abaissa pour examiner les courbes du relief et traça les trigrammes Qian et Kun, fixant la voie de l'Homme. Alors le peuple s'ouvrit à l'intelligence. Il fut conscient des liens d'affection entre père et fils, des devoirs qui obligent princes et sujets, des

être indifférent [...]. Un fils pieux sert son père au lieu de l'évincer; un sujet loyal, loin de chercher à s'emparer du trône, s'emploie à servir le prince. » (chap. LI : 541). Voir aussi XXXLI, p. 411 ; XLVI, p. 487.

19. Yantielun, XII, 5, trad. Levi (2010): 71.

20. Yantielun, LVIII, 4, trad. Levi (2010): 297.

21. Zhuangzi, XII, trad. Levi (2006): 104.

22. Wenzi, X, 12f. 
distinctions qui existent entre les sexes et de la hiérarchie qui sépare aînés et cadets. Par la suite les cent fonctions furent instaurées et la Voie royale fixée ${ }^{23}$.»

Certes, c'est l'ensemble des relations sociales qui se voit définir grâce à l'établissement des deux symboles hexagrammatiques, bien qu'elles dérivent toutes de la relation père-fils, soubassement de la Voie royale dans la mesure où elle fournit la norme de la subordination du sujet à son roi, lequel exemplifie en retour l'autorité et la sollicitude paternelles. Il revient à Zhuangzi le mérite ambigu d'énoncer la règle dans sa généralité abstraite, à la façon d'un impératif catégorique, par la bouche de Confucius rappelant son ami, Gao, duc de She, à ses devoirs de vassal: «Il est sur terre deux prescriptions fondamentales. L'une est dictée par l'instinct, l'autre par la société. L'amour des enfants pour leurs parents est de l'ordre de l'instinct, aucune force au monde ne peut le leur enlever du cœur; le dévouement du sujet à l'égard du souverain ressortit au devoir social. Où qu'il soit, il lui est soumis. Telles sont les deux obligations essentielles auxquelles il est impossible de se soustraire. Un fils vraiment pieux obéit à ses parents avec joie en toutes circonstances; un sujet qui pousse la loyauté jusqu'au bout sert son seigneur en acceptant avec bonne grâce toutes les missions dont il lui plaît de le charger. Qui possède une vertu parfaite, ne pensant qu'à servir parents et prince de tout cœur, ne se laisse pas ébranler par les émotions et, sachant que c'est là quelque chose contre lequel il ne peut rien, il s'abandonne sereinement à cette fatalité. Nul ne peut échapper aux devoirs inhérents à sa qualité de fils ou de sujet ${ }^{24}$. » Même si le texte prend soin de distinguer les deux formes d'obligation, l'une étant de nature instinctuelle et l'autre éthique, elles n'en sont pas moins équivalentes, en ce que la piété filiale est à la nature ce que la loyauté est à la culture. Ou, si l'on préfère, l'obéissance au prince est l'expression sociale d'un tropisme familial.

L'équivalence se situe moins sur le plan de l'autorité et de la loi que de la réciprocité des obligations. Le sujet doit fidélité xin et loyauté zhong à son prince comme tout fils doit se montrer pieux xiao envers son père: «Quand la Voie préside aux rapports entre prince et ministres, la loyauté et la générosité règnent entre eux, aux rapports entre père et fils, l'affection et la piété filiale règnent entre eux », assène le Wenzi ${ }^{25}$ et cela parce que l'un et l'autre ont un impératif moral de don en retour (bao); ils sont des obligés, l'un comme l'autre devant se montrer reconnaissants des bontés et de la sollicitude que le père ou le prince ont manifestées à leur égard. Mais ceci signifie aussi, en vertu de la même loi de réciprocité, que les pères et les souverains ne doivent attendre de

23. Хinyu, I, trad. Levi (2003): 21.

24. Zhuangzi, IV, trad. Levi (2006): 38.

25. Wenzi, $\mathrm{V}, 1 \mathrm{c}$. 
la soumission de la part de leurs enfants et leurs sujets qu'autant qu'eux-mêmes se montrent bons et aimants, en d'autres termes, qu'ils se conduisent en pères et en souverains : «Même un père ne peut demander à un fils qu'il lui manifeste de l'amour quand sa conduite inspire la haine, un souverain ne peut exiger de ses sujets qu'ils lui manifestent du respect quand il ne mérite que le mépris ${ }^{26}{ }$, dira un court traité de la fin des Royaumes combattants, rattaché au courant Huang-Lao. Déjà, à l'occasion de son entrevue au palais des Neiges avec le roi Xuan de Qi, Mencius ne se fait pas faute de rappeler à son royal interlocuteur cette règle d'or: «Si le prince se réjouit des joies de son peuple, son peuple se réjouira de ses joies, s'il se tourmente pour les tourments des autres, son peuple, en retour, se tourmentera de ses tourments. Un souverain qui se réjouit avec tout l'empire et s'afflige avec tout l'empire est appelé à régner sur lui ${ }^{27}$.»

Il va de soi que cette générosité et cette sollicitude n'ont rien d'intéressé; elles sont le mouvement spontané de l'âme dicté en quelque sorte par l'essence même de la paternité et de la royauté. Comme l'affirme non sans emphase un développement du Huainanzi : «Un père aimant prodigue son affection à son fils non parce qu'il en attend de la reconnaissance, mais parce qu'aucune force au monde ne saurait la lui arracher du cœur; un sage monarque veille sur son peuple, non dans l'espoir d'en tirer avantage, mais parce que cette sollicitude fait partie de sa nature et que rien ne saurait la $\operatorname{tarir}^{28}$.»

Le même ouvrage, dans la section consacrée à la guerre, montre en même temps tous les fruits que peut apporter sur le plan de l'efficacité pratique une telle sollicitude paternelle de la part du souverain:

Ne pas avoir le cœur serré quand son peuple se noie n'est pas le fait d'un sage souverain. Il suffit, en effet, que les supérieurs traitent leurs subordonnés comme des fils pour que ces derniers considèrent leurs supérieurs comme des pères; que les supérieurs traitent leurs subordonnés comme des frères cadets et ceux-ci les aimeront comme des frères aînés. Qui traite ses inférieurs comme des fils régnera sur le monde; des inférieurs qui vénèrent leurs supérieurs comme des pères assureront le gouvernement parfait dans l'empire. Qui traite ses inférieurs comme des frères cadets n'aura aucune peine à les faire mourir pour lui ; quand les subordonnés aiment leurs supérieurs comme des frères aînés, ceux-ci en retour leur sacrifieront tout sans hésiter. Toute guerre contre une armée où tous les combattants sont des pères et des fils ou des frères aînés et des frères cadets est vouée à l'échec ${ }^{29}$.

26. Huangdi sijing, III, «Aphorismes», trad. Levi (2009): 218.

27. Mencius, IB 4.

28. Huainanzi, X, 4a.

29. Huainanzi, XV, 18a-b. 


\section{Jean Levi}

Nous aurions là, finalement, la confirmation, tant sur le plan des représentations idéologiques que de la phraséologie véhiculée par le discours politique, de l'intuition de Max Weber sur l'essence de l'organisation bureaucratique chinoise. Pour le sociologue allemand, dont les études sur les sociétés traditionnelles orientales et extrême-orientales doivent fournir la contre-preuve de la validité de ses thèses sur l'esprit du capitalisme et l'éthique protestante, le régime impérial n'est que la projection, à l'échelle de la société tout entière, de l'organisation familiale patriarcale. La relation père-fils se donne pour la forme paradigmatique du rapport prince-sujet, lequel modèle toutes les relations hiérarchiques de supérieur à inférieur et l'équation prince = père est la pierre angulaire de l'édifice social.

Dans ce système, la relation familiale père/fils, dans la mesure où elle sert de soubassement à la relation politique prince/sujet, prime sur elle, en sorte que tout sujet doit s'acquitter de ses devoirs de fils pieux avant ses obligations de loyal serviteur du souverain. Mieux, il ne saurait y avoir de véritables conflits de devoirs, car être un bon sujet, c'est avant tout être un bon fils. Le dévouement aux parents forme l'ossature de l'ordre social. Comme le dit Liang Shuming dans les Idées maîtresses de la culture chinoise: «En Chine la piété filiale et le respect des aînés sont censés assurer naturellement l'ordre dans l'Empire; et le souverain pense que rien ne vaut la piété filiale pour conduire l'Empire ${ }^{30}$.» En cela il ne fait que reprendre les vues lumineuses de Montesquieu: «Le respect pour les pères était nécessairement lié à tout ce qui représentait les pères: les vieillards, les maîtres, les magistrats, l'empereur. Ce respect pour les pères supposait un retour d'amour pour les enfants; et par conséquent le même retour des vieillards aux jeunes gens, des magistrats à ceux qui leur étaient soumis, de l'empereur à ses sujets. Tout cela formait les rites, et ces rites l'esprit général de la Nation ${ }^{31}$.» Et il conclut: «Cet empire est formé sur le gouvernement d'une famille. Si vous diminuez l'autorité paternelle, ou même si vous retranchez les cérémonies qui expriment le respect que l'on a pour elle, vous affaiblissez le respect pour les magistrats, qu'on regarde comme des pères; les magistrats n'auront plus le même soin pour les peuples, qu'ils doivent considérer comme des enfants; ce rapport d'amour qui est entre le prince et ses sujets se perdra aussi peu à peu. Retranchez une de ces pratiques et vous ébranlez l'État ${ }^{32}$.»

30. Liang Shuming (1970): 84-85 et, pour la traduction française, Masson (2010): 115.

31. De l'esprit des lois, XIX,19; voir Montesquieu ([1748] rééd. 1973): I, 341 (cité par Liang Shuming, p. 85 et 116 de la traduction française).

32. Montesquieu ([1748] rééd. 1973): 341. 
Le père insuffisant. Remarques sur la phraséologie politique en Chine ancienne

\section{Le souverain comme père et mère du peuple}

De nombreux chercheurs ont dénoncé, ces dernières années, le caractère schématique des analyses wébériennes concernant le caractère purement patriarcal de la société chinoise et la prévalence de la seule relation père-fils; ils ont cherché à fournir des éléments précis permettant de nuancer la vision caricaturale d'une civilisation chinoise entièrement façonnée par la norme paternelle et l'ordre hiérarchique. Des savants ${ }^{33}$, pour des époques très différentes, ont montré que les deux pouvoirs ne sont nullement équivalents ; l'autorité paternelle n'est pas la traduction dans la sphère réduite de la famille de la souveraineté impériale ou royale. Tout d'abord parce que, à la différence du pater familias romain, le père de famille chinois n'a pas pouvoir de vie et de mort sur les membres de sa maisonnée, puisque les affaires domestiques sont du ressort de l'État ${ }^{34}$. Et d'autre part, parce qu'à la différence de la relation père-fils où les rôles sont mobiles, tout père ayant été fils et tout fils étant destiné, en principe, à devenir père à son tour, aucun sujet, en dehors du fils aîné du monarque, n'est habilité, à moins d'une usurpation, à exercer le pouvoir souverain. Il ne s'agit donc pas d'une égalité où les deux termes de l'équation seraient interchangeables, mais d'une relation univoque où la comparaison ne vaut que dans un seul sens ${ }^{35}$. Si le souverain est un père pour son peuple, les pères ne sont pas des souverains dans leur maisonnée, ou ne le sont que partiellement. En outre, il ne fait guère de doute que la dimension maternelle, par exemple, comme les liaisons horizontales tissées par l'amitié ou les rapports d'égalité entre collègues jouent un rôle fondamental comme ciment de l'édifice social, et qu'ils ont été trop souvent occultés même dans les études récentes en raison de la pétition de principe de la primauté de l'ordre paternaliste et hiérarchique. Miranda Brown, dans une étude extrêmement fouillée, s'emploie à battre en brèche les clichés concernant la piété filiale. Elle montre entre autres que, bien que l'obligation du deuil de trois ans pour un parent soit impérative et que le crime d'impiété filiale (buxiao) soit puni de la peine de mort, cette prescription n'était pourtant que très rarement respectée. La contradiction s'expliquerait selon elle par une conception des devoirs filiaux chez beaucoup de membres de l'élite qui s'écarte du modèle wébérien de la stricte homothétie des codes familiaux et politiques. En effet, le culte rendu au père se subsume au dévouement à la communauté. Pour une

33. Lewis (2006) : 77-133; Nylan (1996): 1-16; Hamilton (1986) : 393-445.

34. Ainsi que le manifestent aussi les documents législatifs d'époque Qin exhumés d'une tombe à Shuihudi.

35. C'est ce que le Guanzi exprime de façon la plus nette: «Quand on est fils, remplacer son père est chose conforme et juste. Quand on est ministre, prendre la place de son prince est une usurpation. Voir Graziani (2011): 67. 


\section{Jean Levi}

portion importante de l'aristocratie lettrée, la meilleure façon d'honorer ses parents est de s'illustrer au service de la Nation, la gloire des fils rejaillissant sur les aïeux et contribuant à la pérennité de leur mémoire. Les liens personnels qui attachent un sujet au souverain comme un fils à un père se transforment dès lors en une obligation abstraite et impartiale de se dévouer corps et âme aux affaires du pays; à telle enseigne que non seulement l'adéquation du père au prince se trouve mise à mal, mais que devoirs au père et devoirs au Prince - sous la forme abstraite de l'État - peuvent se révéler antagonistes. Mais c'est surtout par un autre biais que l'auteur ébranle le modèle standard de l'adéquation pèresouverain; il s'agit cette fois-ci de la primauté de la relation maternelle. Une étude précise et serrée de la phraséologie des inscriptions funéraires élevées à la demande d'un endeuillé et d'autres sources traitant de cette question sous les Han postérieurs révèle de façon inattendue que le deuil de la mère y est un thème tout aussi prisé que celui du père; c'est ainsi que la relation mère-fils vient doubler la relation père-fils, et même parfois la supplanter. Les liens maternels apparaissent comme le contrepoids affectif et le pendant intimiste, relevant de la sphère privée, à la relation paternelle, dès lors assimilée à la sphère publique et sociale. On pourrait dire, pour reprendre une terminologie plus chinoise, que certains membres de l'appareil d'État identifiant la relation à la mère avec la «reconnaissance», en, suscitée par la vertu cardinale de ren, «bonté », et celle au père avec le respect, jing, obtenu par l'exercice de la rigueur, yi, vont donner la préséance à la sphère intime et personnelle, dans la mesure où, dans l'échelle traditionnelle des vertus, le ren, la bonté, prime le yi, la justice. Ce faisant, l'une des prémisses fondamentales de l'adéquation de la famille et de l'État, à savoir la primauté de l'ordre paternel, se trouve dès lors remise en question. Toutefois, Miranda Brown oppose de façon trop statique et figée la sphère maternelle à la sphère paternelle comme représentatives de deux domaines inconciliables. En réalité, la sphère maternelle se trouve déjà intégrée à l'espace public et masculin, tout au moins au niveau de la phraséologie. Sous les Royaumes combattants ( $\mathrm{V}^{\mathrm{e}}$-III ${ }^{\mathrm{e}}$ siècle av. J.-C.), l'idéologie lettrée s'était employée à gagner à la sphère politique la dimension féminine et maternelle à travers la métaphore, non pas d'un prince père, mais d'un prince «père et mère».

Certes, déjà dans des poèmes du Livre des odes, un souverain peut être qualifié de «père-et-mère du peuple ${ }^{36}$ »; mieux, dans «La grande harangue»

36. L'expression est employée à deux reprises: Shijing, «Daya», chant VII, « Xiongzhuo », (trad. Couvreur (1951): 364) dont il sera question plus loin, et Shijing, II, Xiaoya, II, 7, «Nanshan you tai»: «Sur la montagne au sud pousse l'ailante et au nord le prunier. Prince aimable et distingué vous êtes le père et la mère du peuple; prince aimable et distingué, puisse le souvenir de votre vertu se perpétuer à jamais.» (trad. Couvreur: 195). 
qui ouvre les «Annales de la dynastie des Zhou», la quatrième section du Livre des documents, le roi Wu s'adresse à ses troupes rassemblées au gué de Meng en usant du binôme pour définir le rôle du sage souverain qu'il apparie dès lors non plus au Ciel tout seul, mais au couple formé par le Ciel et la Terre: «Le Ciel et la Terre sont le père et la mère des dix mille êtres. Des dix mille êtres, seul l'homme est doué de raison. Et celui qui se distingue de tous les autres par son intelligence est le Souverain. Ainsi donc le Souverain est le père et la mère du peuple ${ }^{37}$.» Toutefois, ainsi que le note Léon Vandermeersch, si les harangues consignent des matériaux anciens, il est probable que l'expression a été introduite par les compilateurs de la fin des Zhou ${ }^{38}$. Dans l'ode de la section «Daya» du Shijing, le dissyllabe se trouve modulé ou mieux décliné dans les deux autres couplets par d'autres formules binomiales et le souverain devient, après avoir été père et mère du peuple, un refuge (you gui) puis un havre (you $x i)$ pour le peuple. En sorte qu'il n'y a pas d'élaboration conceptuelle autour du terme, comme cela est le cas dans la littérature plus tardive. Elle intervient dans un sermon adressé par le musicien Kuang sur l'art de gouverner à son maître le roi de Jin, consigné dans le Zuozhuan. Comme le prince de Jin s'indigne que les habitants de la principauté de Wei aient eu l'audace de chasser leur prince, le sage lui répond: "N'est-ce pas plutôt le prince qui a exagéré? Un souverain vertueux récompense les bons et châtie les méchants. Il choie ses sujets comme ses propres enfants. Il les couvre comme le Ciel couvre; il les porte comme la Terre porte. Aussi ses sujets sont-ils heureux de le servir; ils l'aiment comme leurs pères et mères, le vénèrent comme le soleil et la lune, le respectent comme les esprits, le craignent comme la foudre ${ }^{39}$.»

Le discours du chef des musiciens, Kuang, est placé par l'auteur du Zuozhuan à la $14^{\mathrm{e}}$ année du duc Xiang, soit en 559 av. J.-C. Mais il ne fait guère de doute que ce sont ses propres vues cosmologisantes qu'il met dans la bouche de Kuang et que la description de la vertu royale qui nous est brossée porte la marque de l'idéologie de la seconde période des Royaumes combattants, quand il s'est agi d'asseoir la légitimité impériale non plus sur le fonctionnement réel des cultes, mais sur un principe transcendant.

Dans un dialogue entre Confucius et Zixia figurant dans un manuscrit sur lattes de bambou de la fin du IV siècle avant notre ère et qui a son parallèle dans le Livre des rites (Liji) et les Propos de l'école de Confucius (Kongzi jiayu), les vers du poème du Livre des odes (Shijing) où il est question d'un «prince amène

37. Shujing, IV, «Taishi», 1, trad. Couvreur (1999): 172.

38. Vandermeersch (1977-1980): II, 191, n. 51.

39. Zuozhuan, 14 e année du duc Xiang; Couvreur (1951): II, 308. 


\section{Jean Levi}

et fraternel, père et mère du peuple ${ }^{40} \gg$ sont prétexte à un long développement sur la nécessité pour un roi de cumuler les deux rôles. À son disciple Zixia qui lui demande à quoi doit s'employer un prince pour mériter le qualificatif dont use le Livre des odes, Confucius répond : «Il faut qu'il remonte à la source des rites et de la musique, qu'il atteigne aux "cinq extrêmes", mette en pratique les "trois sans" et les propage partout à travers l'empire. Il doit par ailleurs savoir par avance quand un malheur va s'abattre sur une des quatre contrées. Ce n'est qu'à cette condition qu'il est le père et la mère du peuple. » Le texte poursuit en fournissant des explications sur la signification des deux formules elliptiques les «cinq extrêmes » et les «trois sans »; les premières, permettant de remonter à la source des sentiments, fournissent la clé du bon gouvernement, lequel repose sur la ritualité, ritualité qui n'est rien d'autre que l'expression et le prolongement de la nature humaine: «Les Odes sont la pointe extrême de l'intention, les Rites la pointe extrême des Odes, la musique la pointe extrême des rites, la commisération la pointe extrême de la musique. Musique et commisération s'engendrent l'une l'autre.» Quant aux «trois sans», il s'agit de la musique sans notes, du rite sans décorum et du deuil sans vêtements mortuaires. Grâce à l'entremise de vers du Livre des odes, Confucius fait comprendre au disciple - et l'auteur au lecteur - ce qu'il faut entendre par ces trois antinomies : la première signifie que le prince gagne la confiance de ses sujets par des actes et non des discours ${ }^{41}$; la seconde, que l'autorité émane d'un maintien digne et irréprochable, et non d'un déploiement de faste ou de manifestations de force ${ }^{42}$; la troisième, que la compassion qu'il manifeste pour les malheurs de ses sujets lui attachera leurs cœurs $^{43}$. En un mot, c'est dire, en les transposant sur le plan plus abstrait et plus général des règles cérémonielles, que le prince doit conjuguer les qualités d'un père et d'une mère pour se faire respecter et aimer du peuple, et parvenir ainsi à lui inculquer le sens des rites. Le passage est à rapprocher d'un autre traité recueilli dans le Livre des rites, «Biaoji ${ }^{44}$ ( «Modèles»), qui s'appuie sur les mêmes vers du Livre des odes pour développer des vues comparables, mais en restant cantonné à la métaphore parentale - ou tout au moins en ne sortant pas du cadre de la division sexuelle des tâches: «Le maître a dit: "Oh que pratiquer

40. Shijing, «Daya», chant VII, « Xiongzhuo», trad. Couvreur: 364.

41. Shijing, Zhousong, VI, trad. Couvreur: 422.

42. Shijing, I, «Guofeng», III, «Beifeng», 1/3, trad. Couvreur: 28.

43. Shijing, 10/4, trad. Couvreur: 38 .

44. Pour la transcription et l'analyse du passage, $c f$. Fang Xudong (2004): «Shangbojian "min zhi fumu" bian lunxi », in Shangboguan zang zhanguo chu zhujian yanjiu (xubian). Shanghai: shudian chubanshe, p. 257-276. Le passage parallèle du Liji figure dans le chapitre XXVI «Kongzi xianju» (Confucius en son particulier), Couvreur: II, 391-399; et Kongzi jiayu, VI, 27, «Lun li». 
la bonté, telle que la conçoit l'homme de bien, est difficile !" Le Livre des odes dit: "Ce prince accommodant et fraternel est le père et la mère du peuple." Accommodant, il sait contraindre le peuple à l'étude; fraternel, il lui apporte la joie et la paix. En sorte que la musique ne favorise pas la licence ni les rites n'étouffent l'affection; il est rassurant quoique sévère; respecté quoique prévenant et débonnaire. Le peuple le révère comme un père et le chérit comme une mère. Ce n'est qu'à ces conditions que l'on peut être le père et la mère du peuple. Seul celui qui a su développer en lui la Vertu parfaite peut y prétendre. Un père a de l'attachement pour ses fils s'ils sont capables et n'a que mépris s'ils sont des vauriens, tandis qu'une mère a de l'attachement pour ses fils capables et de la compassion pour ses fils incapables. C'est la raison pour laquelle on a plus de tendresse que d'estime pour sa mère et plus de respect que de tendresse pour son père ${ }^{45}$.» Ces deux formes alternatives et contradictoires du rapport parents/enfants se trouvent subsumées en oppositions plus générales régies par la dichotomie yin/yang puisque, en guise de conclusion, le texte range la relation à la mère dans celle que l'on a pour le familier: l'eau, la terre et le destin, tandis que celle au père ressortit au paradigme des choses ou des entités qui inspirent une crainte révérencielle: le feu, le ciel, les dieux. En transposant la relation père/mère dans d'autres antinomies plus radicales et plus abstraites, l'auteur du traité entendait souligner que le bon souverain sait concilier les inconciliables; le tout du prince excède, et de loin, la somme des parties qui le composent, père et mère ; l'incise a donc valeur explicative, elle justifie la conclusion: seul le Saint, l'homme à la Vertu parfaite, peut être père et mère du peuple, car en passant de la sphère privée à la sphère publique s'opère un saut qualitatif. Toutefois, avec le saut qualitatif se produit une déperdition de la réalité du contenu: la double désignation, en se fondant en une instance qui les dépasse, ne peut plus avoir de signification autre que métaphorique. L'expression «Père et mère du peuple» se vide du référent familial pour ne plus désigner que le mode de gouvernement confucéen. Celui-ci se fait fort d'assurer l'ordre social grâce à l'exemple qu'offre le Souverain, identifié au Saint, dont les actes moraux et rituellement corrects, chargés d'une haute valeur éducative, transforment les mœurs du peuple. En sorte qu'en s'adjoignant les vertus d'une mère, le Souverain-Père se fait avant tout Pédagogue suprême. Ainsi la dimension paternelle - et paternaliste - de la royauté s'en trouve tout à la fois exaltée et réifiée, ou tout au moins transmutée dans une abstraction qui la fait disparaître en la transcendant. En devenant plus que père symboliquement, le souverain devient moins qu'un père, car il cesse de l'être pratiquement.

45. Couvreur (1950), Mémoires sur les bienséances, t. 2, XXIX, 28, 29: 496-497. 


\section{Jean Levi}

À partir du Iv ${ }^{\mathrm{e}}$ siècle av. J.-C., le binôme fumu, «père et mère», sert à qualifier le bon souverain de façon quasi automatique et fait office d'épithète homérique. Mencius, dans ses discussions avec le prince Hui de Liang, y recourt sans même se donner la peine d'en expliciter la signification: «Si celui qui est le père-etmère du peuple ne se fait pas scrupule, dans l'exercice de son gouvernement, de livrer les hommes en pâture aux animaux, peut-il encore prétendre à ce titre ${ }^{46}$ ?» Déjà poncif du vivant de Mencius, le terme, perdant ses connotations familiales et affectives, deviendra une simple périphrase visant à désigner tout détenteur de l'autorité, qu'il soit roi ou préfet. Ainsi, à côté du souverain père et ciel du peuple se développe le motif concurrent du souverain père et mère du peuple qu'il couvre et porte comme le Ciel et la Terre.»

Xunzi, le grand penseur confucéen du $\mathrm{III}^{\mathrm{e}}$ siècle avant notre ère, dans un développement fameux dévolu à la Voie du Souverain, répondant à une question sur la nécessité de porter pour des dignitaires le deuil de trois ans à la suite du décès d'un monarque, explique que ce n'est que justice, le prince cumulant dans sa personne les vertus paternelles et maternelles, ce qui lui vaut au demeurant l'appellation de «père et mère du peuple» (min zhi fumu):

Le souverain est le garant de l'ordre, la source de la civilité, l'accomplissement ultime de l'accord entre les sentiments et leur expression. N'est-il pas normal, dans ces conditions, que ses sujets lui rendent les plus grands hommages? On trouve cette strophe dans le Livre des odes: «Ce prince aimable et fraternel est le père et la mère du peuple ${ }^{47}$ ». Ainsi un prince peut être qualifié de «père et de mère». Un père est capable d'engendrer un enfant mais non de le nourrir; une mère est capable de le nourrir mais non de l'éduquer. Or le souverain est à la fois capable de nourrir et d'éduquer son peuple. Trois ans de deuil, est-ce vraiment assez? Une nourrice allaite durant trois mois, une mère aimante s'occupe de vêtir son enfant durant neuf mois; alors qu'un prince cumule ces deux fonctions, trois ans de deuil, je trouve que ce n'est pas de trop ${ }^{48}$ !

Un article d'une des sections des Quatre canons de l'empereur Jaune développe le thème en l'associant à la notion de nécessaire rétribution:

Un père qui n'agit pas en père ne peut espérer que son fils le serve comme un fils; sans la bonté d'une mère, le souverain ne saurait obtenir que le peuple lui consacre le meilleur de ses forces. Ce n'est que lorsqu'on cumule les vertus d'un père et d'une

46. Mencius, IA, 4, trad. Couvreur: 307.

47. Voir pour les références la note 38 .

48. Voir Zhuzi jicheng, II, Xunzi, XIX: 248. Il est probable que Xunzi s'inspire, dans ce passage, du développement du manuscrit de Shanghai «Père et Mère du peuple» recueilli par la suite dans le Liji et le Kongzi jiayu. 
mère que l'on est à même de dispenser les bienfaits du ciel et de la terre. Ce n'est que lorsque ces trois préalables sont réunis que l'on peut mener à bien ses entreprises ${ }^{49}$.

Sous les Han, la formule fleurit dans la bouche des tenants de tous les courants, qu'ils soient légistes ou confucéens. Dans la Dispute sur le sel et le fer, les deux camps ne se font pas faute de se resservir la formule en l'accommodant à la sauce de leur propre doctrine: «Pour un dirigeant, ne pas chercher à rendre lumineux des règlements et des instructions abstrus, ne pas soutenir le peuple quand il trébuche, c'est faire preuve de la même insensibilité qu'un homme qui verrait un nourrisson au bord d'un puits et resterait les bras croisés en attendant qu'il tombe. Un tel prince est-il encore le père-et-mère du peuple ${ }^{50}$ ? font valoir les lettrés, télescopant en une formule plusieurs assertions disséminées dans les péroraisons de leur maître à penser, Mencius, afin de mettre en exergue la nécessaire indulgence d'un véritable monarque. Ou bien alors avec encore plus de pathos: "Lorsque, à la vue de ses sujets mourant de faim, le père-et-mère du peuple s'exclame: "Ce n'est pas notre faute, c'est la récolte", il me fait penser à un criminel qui, après avoir égorgé quelqu'un avec un poignard, accuserait la lame ${ }^{51}$.» Le vice-premier ministre reprend la même image; mais, en mettant l'accent sur le respect plutôt que sur l'affection, il l'utilise à des fins opposées: «Le Fils du Ciel est le père-et-mère du peuple; et celui-ci ne songe qu'à le servir comme son esclave ${ }^{52} \gg$.

Dans la tradition occidentale, le paternalisme se manifeste par l'appellation «père des peuples » ou «petit père des peuples », formule qui s'est attachée tout particulièrement aux tsars de Russie avant de devenir l'apanage de Staline. La fonction paternelle y adhère absolument à l'exercice du pouvoir et se suffit en quelque sorte à elle-même, puisqu'elle fournit son propre antidote débonnaire et paterne à sa sévérité. En Chine, en voulant faire du prince plus qu'un père et en englobant dans sa personne les deux aspects de l'autorité parentale - la fermeté et la douceur -, les idéologues lettrés visaient certes à exalter la figure du souverain, tout en cherchant à tempérer ce que pouvait avoir d'excessif et de brutal l'absolutisme impérial, mais ce faisant, ils confessaient la carence du modèle paternel, puisque celui-ci s'avérait impuissant à exercer à lui seul, avec

49. Huangdi sijing, I, «Le pouvoir régulateur...», trad. Levi (2009): 136.

50. Yantielun, XXXIII, 4, trad. Levi (2010): 183-185.

51. Yantielun, XXXVI, 2, trad. Levi (2010): 194. Ou bien encore: «Le prince veille sur ses sujets comme une mère et un père sur ses enfants. Il ne songe qu'à leur prodiguer ses bienfaits. [...] Que le prince agisse en prince; le vassal se conduira en vassal. Que le père agisse en père et les fils se conduiront en fils! Aurait-on alors besoin d'établir des règlements sur la responsabilité collective et les hommes au pouvoir auraient-ils des charges si lourdes à porter?» (LVII, 3, p. 290).

52. Yantielun, XXXVIII, 3, trad. Levi (2010): 203. 


\section{Jean Levi}

le simple concours de ses vertus, la régulation de l'ensemble du corps social. La phraséologie censée magnifier la perfection et la toute-puissance du souverain trahit par la même occasion le divorce entre l'exercice réel du pouvoir et sa représentation.

\section{La dialectique de la poule et du coq}

Toutefois, si la dimension féminine et maternelle est récupérée dans la sphère même qui lui semble la plus étrangère, la sphère politique, c'est que les vertus féminines sont devenues éminemment politiques ou plus exactement stratégiques. Cette mutation tient à la dévalorisation des vertus héroïques et viriles qui a suivi les transformations des techniques de combat, lorsque la guerre de masse a remplacé le combat chevaleresque. Le régime de la royauté archaïque qui réglait la société à travers l'embranchement des collèges cultuels, en sorte que l'acte sacrificiel se confondait avec la pratique du gouvernement, est remplacé par un système de gestion autoritaire et centralisé. Aux collèges cultuels imbriqués se substitue une organisation territoriale d'unités administratives à partir de laquelle seront établis les différents systèmes de levées de masse. Ceux-ci conduisent à une militarisation totale de la société au Iv siècle av. J.-C. L'extension de la guerre à toutes les couches de la population aboutit à ravaler le combattant au rang de simple pion dans un dispositif qui le dépasse. La guerre nécessite dès lors les aptitudes dont on crédite le yin, l'emblème du féminin: souplesse, faiblesse, passivité. La société des Zhou était à l'origine une aristocratie guerrière; sitôt que la charrerie noble perd son prestige, c'est l'ensemble des institutions des Zhou qui deviennent obsolètes, ainsi que les valeurs viriles qui les sous-tendaient. Les théoriciens du pouvoir de l'époque des Royaumes combattants, qui en prennent acte, en viennent à penser que toute domination n'est possible qu'à travers un comportement féminin. Qu'en un mot, pour être coq, il faut savoir être poule. Un passage d'un manuscrit de la tradition de l'empereur Jaune porte le titre significatif de «Conduites de la poule et du coq». Il y est dit en substance:

Arrogance et superbe : voilà l'attitude masculine; mesure et retenue: comportement féminin. Qui fait le coq dilapide, qui fait la poule thésaurise. Acquérir par l'attitude du coq, ce n'est pas s'assurer la fortune, perdre par la conduite de la poule, c'est gagner des dividendes. Succès répétés par le coq, malheurs redoublés, les tracas succèdent aux échecs jusqu'à la ruine finale. Échecs répétés par la poule, gains accumulés. En veillant à respecter cette règle, les profits sont extrêmes. Tous les malheurs viennent de ce que celui qui se met en avant est désavantagé par rapport à celui qui se tient en 
retrait. Par l'attitude de la poule on pourra se mettre en avant sans connaître le malheur. Par l'attitude masculine on connaîtra des revers même en se tenant en retrait ${ }^{53}$.

Les mêmes thèmes sont repris dans un autre paragraphe intitulé : «La voie de la soumission». On remarquera que la soumission est la vertu cardinale de la femme. Mais cette voie féminine permet d'accéder à la toute-puissance. L'empereur Grande-Cour, qui parvint à étendre son emprise sur l'univers tout entier, «se conformait à l'attitude féminine afin de favoriser en lui la souplesse $^{54} \gg$. Car le comportement féminin ne vise malgré tout à rien d'autre qu'à réaliser les idéaux masculins de triomphe et de domination: "Combattre sans s'exposer à la vengeance, prendre des territoires sans susciter de résistance, remporter des victoires au-dehors tout en s'enrichissant au-dedans, faire resplendir son renom aux moindres frais, tels sont les résultats que l'on peut attendre de la voie de la soumission ${ }^{55}$.»

Dans le Wenzi, l'attitude de la poule est appariée à l'action du Ciel :

Le Saint se conforme à l'attitude de la poule; il proscrit toute superbe et n'ose adopter un comportement brutal. Prenant l'attitude de la poule, il peut devenir coq; n'osant faire montre de superbe, il subsiste longtemps... Grâce à l'attitude féminine, il progresse chaque jour et ses mérites ne connaissent jamais d'éclipses, à l'image de la marche du Ciel. Il est dans les sentiments humains d'aspirer à une position élevée plutôt que basse, à des gains plutôt qu'à des pertes, à des avantages plutôt qu'à des désagréments, aux honneurs plutôt qu'à l'opprobre, à la noblesse plutôt que la roture. La plupart des gens échouent de se démener ainsi et n'obtiennent rien du tout à force de tout vouloir. Le Saint, prenant modèle sur le Ciel, réussit puisqu'il n'agit, obtient puisqu'il n'étreint ${ }^{56}$.

Les textes cosmogoniques et médicaux opposent la passivité (jing ${ }^{57}$ ) de la femelle à l'activité (zao) du mâle. Le yin est caché, secret (you), le yang est lumineux (ming). Il se dévoile et donne à voir. Et par là même il donne prise. C'est la même attitude de repli, de fermeture propre aux femmes confinées dans

53. Huangdi sijing, II, «L'attitude de la poule et du coq», trad. Levi (2009): 193-195.

54. Huangdi sijing, II, «La voie de la soumission», trad. Levi (2009): 207.

55. Huangdi sijing, II, «La voie de la soumission», trad. Levi (2009): 208. Le Huainanzi reprend cette thèse en lui donnant une dimension métaphysique: «Celui qui a obtenu le Tao met tous ses soins à se montrer faible et pourtant il fait preuve de sa force. Il vide son cœur et pourtant il réagit à propos... C'est pourquoi celui qui veut être fort doit préserver cette force par la faiblesse... Voilà pourquoi le Saint s'en tient à la voie de la pureté et se conduit comme une poule. Il se plie à ce qu'il convient, répond aux circonstances. Il est derrière, jamais devant, ployant et faible il demeure calme et stable dans la paix et la passivité» (Huainanzi, I: 14b-15a).

56. Wenzi, III, «Jiushou»: 11-12.

57. Dans le glossaire, voir jing (2). 


\section{Jean Levi}

le gynécée et dont la saison est l'hiver, la morte-saison qui appartient aux tisserandes recluses dans leur demeure, que conseillent les traités de gouvernement. Le Huainanzi donne ces conseils au prince:

Reste sur la défensive et veille sur l'intérieur, ferme-toi comme une huître sans te tourner vers le dehors, car trop de savoir nuit. Regarde comme une mère, écoute comme une mère. Garde ton esprit dans la passivité et ta forme sera parfaite. Car personne n'a pu conquérir le «cela» sans conquérir son moi ${ }^{58}$.

À travers la chaîne syntagmatique du discours politico-stratégique propre aux Royaumes combattants, se dessinent les connotations paradigmatiques du cadre référentiel masculin/féminin ou, mieux, père/mère, sur tous les plans et à tous les niveaux, mais détournés, subvertis par rapport à la norme confucianiste et patriarcale. Le thème récurrent de l'eau, blason féminin par excellence, y fait éclater le caractère paradoxal de l'inefficience masculine et paternelle. Dans le Huainanzi, l'eau est élevée à la dignité de principe primordial: «Le sans-forme est le patriarche de toute forme, l'inaudible l'ancêtre de l'audible. Ils ont pour fils Lumière, et Eau pour petite-fille, tous deux issus du sans-forme [...]. L'eau se peut diriger mais non détruire. C'est pourquoi dans le monde des images rien n'est plus noble que l'eau, surgissant dans la vie, disparaissant dans la mort. Allant de l'être au néant et du néant à l'être, faible et basse ${ }^{59}$.»

Ses propriétés l'élèvent en effet au statut d'entité divine au même titre que le Ciel et la Terre:

L'eau est la plus molle et la plus faible des choses et pourtant son immensité est sans borne, sa profondeur insondable... Elle embrasse la foule des créatures dans un même amour; ses largesses se répandent jusqu'au plus humble des insectes sans rien exiger en échange. Coulant en un flux inépuisable, elle est pourtant si ténue que l'on ne peut la saisir dans la main. On la transperce sans la blesser, la coupe sans la scinder, la brûle sans l'enflammer. Faible et fuyante, elle se fraie son chemin en épousant le relief sans pour autant disparaître ou se disperser... Elle pourvoit aux besoins de tous les êtres sans en favoriser aucun et ignore la distinction entre privé et public. Elle s'unit au ciel et à la terre dans une même immensité. C'est ce qu'on appelle la Vertu suprême. Si l'eau s'accomplit en tant que Vertu suprême, c'est qu'elle est faible et fuyante ${ }^{60}$.

Tous ces textes portent la marque du Laozi, quand ils n'en sont pas une glose ou une paraphrase. Celui-ci se présente comme un hymne à la gloire de la féminité - et peut-être plus encore de la maternité, comme le prouvent maints et maints

58. Huainanzi, XIV, 4a.

59. Huainanzi, I, 16a.

60. Huainanzi, I, 17a. 
versets, dont nous ne citerons que quelques-uns pour mémoire: «Le monde a une origine: je l'appelle la mère du Tao. Qui étreint la Mère connaît le Fils; qui connaît le Fils saura garder la Mère et sera toujours à l'abri du malheur ${ }^{61}{ }$; «Une grande nation est le cours inférieur d'un fleuve, le point de rencontre de l'univers, la femelle de la création. La femelle, par sa passivité, aura toujours raison du mâle ${ }^{62} » ;$ «L'esprit du Val ne meurt point, c'est la Sombre Femelle. La porte de la Sombre Femelle est la racine de la Terre et du $\mathrm{Ciel}^{63}{ }^{3}$; « «Connais le masculin, garde le féminin; sois le ravin du monde. Qui est le ravin du monde s'attache à jamais la Vertu ${ }^{64} \gg$. L'eau n'en est que le symbole le plus prestigieux : «L'eau bénéfique à tous n'est rivale de rien. Elle se trouve là où la foule ne va point ${ }^{65}$.» On voit donc que, grâce à un retournement total de toutes les valeurs traditionnelles poussées à leur point ultime, la réflexion sur l'autocratisme paternaliste conduit non seulement à affirmer la nécessaire complémentarité des vertus paternelles et maternelles, mais encore à évincer les premières au profit des dernières. Le souverain se fait mère poule; non pas tellement en ce qu'il veille avec amour sur son peuple mais en ce que cette féminisation lui permet d'accoucher de la domination absolue. Toutefois, s'il y a chez Laozi une exaltation de la féminité, tout au moins sous les espèces de la maternité, les développements plus tardifs de l'école Huang-Lao se montrent autrement plus ambigus quant à la supériorité des valeurs féminines. Celles-ci n'ont de prix que comme moyens, on serait presque tenté de dire comme expédients permettant d'asseoir un pouvoir qui se doit d'être masculin pour rayonner et imposer le respect. Une des sections des appendices du manuscrit $\mathrm{B}$ du Laozi exhumé à Mawangdui assimilé à un ouvrage perdu, le Huangdi sijing, Les Quatre Canons de l'empereur Jaune, se clôt sur une énumération de paires d'objets ou d'attitudes opposés, répondant aux deux rubriques yin et yang et où l'entité yang a toujours la prééminence sur sa contrepartie yin: ainsi le ciel est-il yang, la terre yin; le jour yang, la nuit yin; un pays puissant yang, faible yin; le souverain est yang, le sujet yin; le supérieur yang, l'inférieur yin; l'homme yang, la femme yin; la réussite yang, la misère yin, etc. Et le texte de conclure: «Tout ce qui est yang a le Ciel pour modèle, yin la Terre... Les qualités de la Terre sont la placidité, la lenteur, la rectitude, le repos; son attitude est la souplesse et sa

61. Laozi, LVII. Voir aussi XXIV: «Il était quelque chose d'indistinct, né avant Ciel et Terre, obscur et trouble, solitaire, se suffisant à lui-même, soustrait aux transformations. Je le tiens pour la mère de l'univers. Ne connaissant pas son nom je l'ai prénommé "Voie". »

62. Laozi, LXI.

63. Laozi, VI.

64. Laozi, XXVIII.

65. Laozi, VIII. Voir aussi LXI: «Rien de plus faible que l'eau. Rien ne la surpasse pour entamer le dur et le fort.» 
préoccupation essentielle la stabilité. Elle donne sans jamais contester. Telles sont les lois qui régissent la Terre et tel est le comportement féminin ${ }^{66}$.» Or, dans les deux paragraphes intitulés «L'attitude de la poule et du coq» et «La voie de la soumission» de la section III de ces écrits Huang-Lao, c'est justement cette attitude humble et soumise qui permet à celui qui l'adopte d'avoir le dessus sur celui qui opte pour la politique virile de l'éclat et de la force. Un développement du chapitre inaugural du Wenzi, qui offre de fortes affinités avec la quatrième section des Quatre canons, opère lui aussi le même renversement dialectique, même si les objets ne sont pas répartis entre les deux emblèmes yin et yang, mais entre ce qui en semble être une modulation socio-ontique: l'ayant-nom et le sans-nom. Quoi qu'il en soit, là encore les qualités subalternes et inférieures se révèlent être des facteurs de supériorité: «Ce qui est large et épais a nom, ce qui est étriqué et mince est sans nom; ce qui a nom est noble, ce qui est sans nom est vil; la richesse a nom: ce qui a nom est considéré et courtisé ; la pauvreté est sans nom: ce qui est sans nom est méprisé et dédaigné. Le masculin a nom: ce qui a nom est éclatant et glorieux; le féminin est sans nom: le sans nom est obscur et discret; l'abondance a nom: ce qui a nom est porté aux nues; l'insuffisance est sans nom: ce qui est sans nom est ravalé plus bas que terre. Qui a du succès a nom, qui n'en a pas est sans nom. L'ayant-nom est issu du sans-nom, le sans-nom est la mère de l'ayant-nom ${ }^{67}$. » Le but visé est bien entendu le renom et la gloire (lesquels ressortissent aux catégories masculines propres à tout pouvoir patriarcal), mais il ne peut être atteint que par leur négation. D'où ce paradoxe que seules les modalités du yin sont à même d'assurer l'avènement de la puissance paternelle. Dans une conclusion qui n'est pas sans évoquer, par le renversement incessant des contraires les uns dans les autres, la dialectique négative de Theodor Adorno et de Max Horkheimer, l'auteur des «Aphorismes» aboutit à ce paradoxe qu'il faut être sans-nom pour avoir nom. Autant dire qu'il faut être mère pour être père: «Qui est maître du Tao en détient l'Efficace, qui détient l'Efficace remporte des succès, qui a des succès se fait un nom. Qui a nom fait retour au Tao. Qui sait préserver succès et nom est à l'abri du malheur sa vie durant. Les ducs et les rois ont succès et nom; la veuve et l'orphelin n'ont ni succès ni nom. C'est pourquoi il est dit: "Les souverains se donnent du veuf, de l'orphelin, faisant retour à la racine et leur œuvre accomplie, ne s'en prévalent pas"; ayant des succès ils sont bénéfiques, demeurant sans nom, ils contribuent au fonctionnement des choses ${ }^{68}$.» Certes,

66. Le Lao-tseu suivi des Quatre Canons de l'empereur Jaune, III, «Aphorismes», trad. Levi (2009): 224-225.

67. Wenzi, I, 10d.

68. Wenzi, I, 10d. 
la paternité est reconquise au terme du processus de féminisation du prétendant au trône du dragon, mais il n'empêche, la pensée politique de l'époque, tout au moins en ce qui concerne l'un de ses courants les plus importants, est obligée de faire l'aveu de la dramatique insuffisance du père à assurer l'ordre dans l'empire en se contentant de ses qualités de père. En même temps elle accuse l'écart entre les deux fonctions, celle de chef de famille et celle de chef d'État. Car nul ne demande à un père de jouer à la poule pour assurer l'ordre dans la maisonnée, alors que l'adoption des valeurs et du comportement féminin sont la condition sine qua non pour qu'un prince puisse régner.

\section{Han Fei ou le triomphe en trompe-l'œil du père Fouettard}

Ainsi donc, bien que le régime absolutiste chinois dérive d'un exercice de la souveraineté qui prend appui sur le modèle de la puissance paternelle - en même temps qu'il repose sur une idéologie paternaliste -, il trouve sa justification, chez la plupart des auteurs des Royaumes combattants, dans une phraséologie qui manifeste l'insuffisance du modèle paternel, puisque chez les confucéens le concours de la mère s'avère indispensable, tandis que dans les cercles influencés par le taoïsme, celle-ci vient supplanter l'efficience paternelle, tout au moins sur le plan du concept. À première vue les légistes, et particulièrement Han Fei, semblent aller contre cette tendance. Étant donné la part qu'eurent leurs théories dans la formation de l'empire centralisé, il conviendrait alors de n'attacher qu'une importance relative à ces élaborations conceptuelles propres à certains cercles relativement marginaux visant à donner un tour libéral à une domination absolutiste par le recours à la métaphore maternelle. Han Fei pourfend sans relâche ce préjugé, véhiculé par les doctrines lettrées, qui veut que le souverain abandonne la sévérité du chef de famille inhérente à l'exercice de la souveraineté pour la tendresse qui sied aux mères. Tous les écrits du théoricien du pouvoir autocratique sont traversés par l'exaltation du modèle paternel de gouvernement qu'il oppose à l'indulgence coupable de ces princes abusés par les homélies humanitaristes des confucéens. Pour lui, aucun régime politique ne saurait reposer sur l'affection mutuelle. La réciprocité bao, dans la mesure où elle prend sa source dans les sentiments et les pulsions des individus, n'est pas un principe fiable de gouvernement. L'ordre ne saurait reposer que sur la crainte que le souverain inspire à ses peuples; aussi le modèle paradigmatique d'un bon souverain est-il celui de la puissance paternelle, dans ce qu'elle a de rigoureux et de terrible. Dans un chapitre où il dénonce les inconséquences de théories politiques de son époque, il aura cette forte formule : «Des enfants gâtés par une mère aimante ont de nombreux vices, voilà où mène trop d'indulgence; des enfants battus par un père sévère ont toutes les vertus; tels sont les bienfaits 


\section{Jean Levi}

de la rigueur ${ }^{69}$.» Un siècle et demi plus tard, l'adjoint de Sang Hongyang, défendant la politique répressive menée par le gouvernement de son supérieur sous le règne de l'empereur Han Wu di, reprendra en écho: «Une mère trop indulgente a de mauvais sujets pour fils; une grande famille n'a pas d'esclaves insolents car elle sait manier la trique. C'est une folie que de vouloir employer les méthodes qui font d'un fils un vaurien plutôt que celles qui permettent de mater les esclaves ${ }^{70}$.»

Car à la vérité, le but de tout dirigeant étant en premier lieu d'obtenir l'obéissance de ses subordonnés, le prince n'a que faire des qualités d'une mère qui sait se faire aimer de ses sujets, car ce n'est pas l'amour des sujets pour les bontés du prince qui procure puissance et richesse à un État. Tout au contraire, la survie des nations repose sur cette intransigeance et cette sévérité qui attirent à un père de famille digne de ce nom le respect de ses fils et la soumission de ses femmes : «Un prince éclairé s'emploie à multiplier ses défenses et à alourdir les châtiments ; il fait en sorte que le peuple soit arrêté non par le frein de la probité, mais par la cravache des lois et des interdits. Si l'amour d'une mère est deux fois plus fort que celui d'un père, l'autorité d'un père qui sait se faire obéir de son fils vaut celle de dix mères ${ }^{71}$.» Mais cette exaltation de l'image paternelle n'est qu'un faux-semblant. Le père n'est montré en exemple que dans la mesure où il peut servir dans une stratégie de dévalorisation des qualités maternelles. À peine évoquée, la figure paternelle se trouve renvoyée au néant. Le souverain ne saurait être assimilé à un père, car les liens qui attachent le chef de famille à ses enfants sont d'ordre individuel et affectif. Or un État n'est pas une famille: «Les relations entre un prince et ses ministres sont autrement plus lâches que celles qui unissent un père à son fils ${ }^{72} \gg$, constate Han Fei avec la froide lucidité qui le caractérise. Tandis que les rapports entre les gens au sein de la famille obéissent - et encore, seulement dans une certaine mesure - à l'affection, au sein de la société et dans l'appareil d'État, ils sont dictés par le calcul:

Les rapports entre un prince et ses sujets ne sont pas commandés par l'affection qui lie un père et un fils, ils sont dictés uniquement par l'intérêt et le calcul. Aussi, quand un Prince use des techniques adéquates, ses sujets lui consacrent le meilleur de leurs forces et toute velléité de sédition est étouffée. Mais s'il ignore cet art, ses sujets s'emploient à l'abuser et à nouer des intrigues ${ }^{73}$.

69. Han Feizi jijie, XLVI: 320, trad. Levi (1999), «Les six à rebours » : 489.

70. Yantielun, LVII, 4, trad. Levi (2010): 291-292.

71. Han Feizi jijie, XLVI: 320, trad. Levi (1999), «Les six à rebours», Le Tao du prince: 488.

72. Han Feizi, XIV, trad. Levi (1999): 152.

73. Han Feizi, XXXVI, trad. Levi (1999), «Réfutations I» : 408. 
Han Fei dénonce le caractère factice de la métaphore parentale et s'emploie à montrer, dans un long développement, combien la comparaison avec un patron et ses employés serait plus appropriée: «Si le maître prend soin de nourrir la force de travail de son ouvrier, s'il lui manifeste la sollicitude qu'un père ou une mère montrent à leur progéniture, et si l'ouvrier le paie en retour en le servant avec zèle, c'est que chacun a en tête son profit. D'où il ressort que, dans les rapports de services et d'échanges entre les individus, on s'entend avec le plus parfait étranger, fût-il de Yue, pourvu qu'on y trouve son bénéfice, mais si nul n'y trouve son compte, parents et enfants s'entre-déchirent ${ }^{74}$.»

Si une mère ne vaut pas un père pour se faire respecter au sein de la maisonnée, l'autorité d'un père paraît bien peu de chose comparée à la force contraignante et abstraite de la Loi. En sorte que la figure du père se trouve, en fin de compte, tout aussi dévalorisée que celle de la mère; le modèle paternel, même sous sa forme la plus répressive, dans la mesure où il suppose l'intervention d'un individu concret, foyer de désirs et d'affects, doit être abandonné au profit d'une puissance désincarnée et transcendante: «Un fonctionnaire qui n'éprouve aucun amour pour le peuple et fait appliquer les ordonnances vaut dix mille pères. Une mère aimante, en dépit de toute sa tendresse, n'arrive pas à se faire respecter de son fils, mais avec la crainte, n'importe quel fonctionnaire dirige tout un peuple. Je ne sais preuve plus éclairante de la supériorité de l'efficacité de la rigueur sur l'affection. [...] Ainsi des parents qui débordent de tendresse et dont l'unique préoccupation est le bonheur et le confort de leurs enfants ne s'en font pas obéir, tandis qu'un souverain indifférent qui cherche à tirer le maximum de profit de ses sujets fait respecter ses ordres. Un prince avisé, ayant tiré toutes les conséquences de cette vérité, s'attache à accroître toujours davantage le poids de son prestige en inculquant la crainte à ses sujets par sa sévérité et extirpe de son cœur tout sentiment de compassion ou d'amour ${ }^{75}$. » Pour tout dire, quand il s'agit d'assurer l'ordre et d'amener les fortes têtes à résipiscence, aucun père, si sévère soit-il, ne vaudra un policier: "Imaginons qu'il y ait dans une famille un vaurien que ni les colères de ses parents, ni les reproches des voisins, ni les leçons des éducateurs ne parviennent à corriger. Malgré ces trois excellentes choses que sont la sollicitude des parents, les exhortations du voisinage et la sagesse des anciens, il ne s'est pas amélioré d'un poil. Mais il suffit que le magistrat du district, à la tête d'un détachement de gardes et pourvu d'un mandat officiel, lance une opération de police contre les chenapans, pour que notre mauvais garçon, saisi de crainte, réforme sa conduite et change ses

74. Han Feizi, XXXII, trad. Levi (1999): 325.

75. Han Feizi, XLVI, trad. Levi (1999), «Les six à rebours» : 488. 
habitudes. Il faut se rendre à l'évidence : l'amour des parents est impuissant à inculquer les bonnes manières, et rien de tel pour dresser les chenapans que la peur du gendarme. L'affection excite l'arrogance des hommes; la crainte les rend dociles ${ }^{76}$.» Finalement, le credo de Han Fei n'est nullement le dicton romain «Qui bene amat bene castigat», mais bien plutôt: «Seul qui n'aime pas châtie bien.» La figure du père n'est qu'un moment d'un processus dialectique où elle ne prend sens que dans son propre dépassement. Elle marque une étape dans un mouvement ascendant qui doit permettre à l'État de se soustraire à la sphère de la famille qu'entache l'irrationalité des affects individuels pour accéder à la sphère épurée d'un ordre abstrait, impersonnel et nécessaire, dont le modèle n'est pas l'organisation clanique sous la tutelle des Pères, mais l'ordre inéluctable de la nature dont la loi répressive n'est que la diffraction dans la sphère sociale et humaine. Plus encore peut-être que tous les autres courants, Han Fei manifeste la dramatique insuffisance de la puissance paternelle pour assurer l'ordre dans une société où les trois ordres hiérarchiques avaient cessé de coïncider.

En se vidant de sa substance avec l'effondrement de la société noble, le dispositif cultuel libère l'armature conceptuelle sous-jacente sur lequel il s'est édifié, et celle-ci va servir à la justification idéologique du nouveau système d'organisation centralisé et autocratique. L'administration étatique tend à faire régner dans la société les mêmes lois objectives que celles qui meuvent les planètes et gouvernent l'alternance des saisons. Elle confère, parce qu'elle obéit à une rationalité territoriale qui s'est substituée au dispositif lignager et sacrificiel, à celui qui la dirige un statut qui l'apparie au mouvement même des choses, au Tao qui se caractérise par son absence d'être. Le souverain ne saurait être père, car sitôt qu'il se charge de qualités positives, il se dépouille de sa puissance, son efficience tenant précisément à l'absence de toute détermination. C'est la raison pour laquelle, notoirement insuffisant dans son effectivité concrète, il a pu se voir supplanté par l'image de la mère, laquelle se trouve dotée de qualités négatives, si je puis dire. Aussi doit-on voir dans ce qualificatif du prince «pèreet-mère du peuple» - et non pas seulement «père » comme dans le paternalisme occidental - un aveu ou un repentir. L'insertion de l'image passive de la mère dans une sphère réservée aux vertus et aux comportements masculins est le stigmate de ce divorce. Si elle en marque le constat implicite et traduit une prise de conscience aiguë du fait politique, elle n'a pu combler que sur le plan symbolique la fracture qui s'est ainsi ouverte dans cette unité organique où le rite formait un phénomène social total; d'où sa propension à s'ériger non pas en concept opératoire mais en masque idéologique. Le pouvoir en Chine, de paternel, n'est

76. Han Feizi, XLIX, trad. Levi (1999), «Les cinq vermines » : 515-517. 
Le père insuffisant. Remarques sur la phraséologie politique en Chine ancienne

même pas devenu paternaliste. Il est un système de régulation sociale abstrait et désincarné recouvert du voile de la fausse conscience où la mère est venue en renfort d'un père qui ne pouvait, à lui seul, en cacher le caractère illusoire.

Il va de soi qu'il ne s'agit là nullement d'un schéma chronologique mais d'une progression en quelque sorte structurelle et logique. La théorie de Han Fei ne met nullement un point final à la métaphore familiale et parentale, bien au contraire. Le légisme étant avant tout une théorie critique et prescriptive de l'exercice absolutiste du pouvoir, il ne pouvait servir de travestissement idéologique. À partir des Han, la nature mensongère - ou si l'on préfère fictive - de la métaphore paternelle est occultée grâce à la sacralisation de la piété filiale. Nous aurions là une vérification chinoise de la loi énoncée de la sorte par Feuerbach dans sa préface à L'Essence du christianisme : «Le sacré grandit à mesure que décroît la vérité et que l'illusion croît $^{77}$.»

77. Feuerbach ([1841], 1992): 108. 


\section{BibliographIE}

BILLETER Jean François (1991). La civilisation chinoise - Introduction: L'univers chinois. In Histoire des mœurs, Encyclopédie de la Pléiade. Paris: Gallimard.

Couvreur Séraphin (trad.) (rééd.1950). Mémoires sur les bienséances et les cérémonies. Paris: Cathasia.

Couvreur Séraphin (trad.) (réimpr. 1951). La Chronique de la principauté de Lou. Paris: Cathasia, Les Belles Lettres.

Couvreur Séraphin (trad.) (1896, rééd. 1966). Cheu King. Fac-similé de l'édition Ho kien Fou. Imprimerie de la Mission catholique.

Couvreur Séraphin (trad.) (1897, réimpr. 1999). Les Annales de la Chine. Paris: You Feng. FANG Xudong 方旭東 (2004). Shangbojian «min zhi fumu» bian lunxi 上博簡 《民之父母》編論析. In Shangboguan cang zhanguo chu zhujian yanjiu (xubian) 上博館藏戰國楚竹簡研究 (續編). Shanghai: Shanghai shudian chubanshe.

Graziani Romain (2011). Écrits de maître Guan. Les quatre chapitres de "L'Art de l'esprit». Paris: Les Belles Lettres, «Bibliothèque Chinoise».

Hamilton Gary (1984). Patriarchalism in Imperial China and Western Europe: A Revision of Weber's Sociology of Domination. Theory and Society, $\mathrm{n}^{\circ} 13$ (3).

Feuerbach Ludwig (rééd. 1992). L'Essence du christianisme. Paris: Gallimard, «Tel».

Le Blanc Charles et Mathieu Rémi (dir.) (2003). Philosophes taoïstes, t. 2: Huainan zi. Paris: Gallimard, «Bibliothèque de la Pléiade».

Levi Jean (trad.) (1999). Han-Fei-tse ou le Tao du prince. Paris: Seuil.

Levi Jean (trad.) (2003): Lu Jia. Nouveaux principes de politique. Honfleur: Zurma.

Levi Jean (trad.) (2008). Ho-Kouan-Tseu. Précis de domination par le Maître à la crête de faisan. Paris: Allia.

Levi Jean (trad.) (2006, rééd. 2010). Les Euvres de Maître Tchouang. Paris: Éditions de l'Encyclopédie des Nuisances.

Levi Jean (2009). Le Lao Tseu suivi des Quatre canons de l'empereur Jaune. Paris: Albin Michel, «Spiritualité».

LeVI Jean (2010). Dispute sur le sel et le fer, présentation, traduction et annotations. Paris: Les Belles Lettres, «Bibliothèque chinoise».

Levi Jean (2012). Écrits de Maître Wen (Wenzi). Paris: Les Belles Lettres, «Bibliothèque chinoise».

LewIs Mark Edward (2006). The Construction of Space in Early China. Albany, NY: State University of New York Press.

LiANG Shuming 梁漱㵓 (1970). Zhongguo wenhua yaoyi 中國文化 要義 Taibei: Zhengzhong shuju. Trad. Michel Masson (2010), sous le titre Les Idées maîtresses de la culture chinoise. Paris: Cerf.

Montesquieu (1748, rééd. 1973). De l'esprit des lois. Paris: Garnier.

Nylan Michael (1996). Confucian Piety and Individualism in Han China. Journal of the American Oriental Society, $\mathrm{n}^{\circ} 116$ (1).

VANDERMEERSCH Léon (1977-1980). Wangdao ou la Voie royale. Recherches sur l'esprit des institutions de la Chine archaïque. Paris: École française d'Extrême-Orient.

WANG Xianshen 王先慎 (dir.), (1998 repr.). Han Feizi jijie 韓非子集解. Beijing: Zhonghua shuju. 
Le père insuffisant. Remarques sur la phraséologie politique en Chine ancienne

\section{Glossaire}

Bao 報

bi 妣

buxiao 不孝

chen 臣

en 恩

$f u$ 父

Guan Zhong 管仲

Houji 后稷

jiao 郊

jing (1) 敬

jing (2) 静

Mingtang 明堂

min zhi fuти 民之父母

Qi Huan gong 齊桓公

ren 仁

xiao 孝

xin 信

$y i$ 义

yin 尹

you gui 做歸

you xi/ji 做㙠

zhong 忠

zhongfu 眾父

$z h o n g f u f u$ 眾父父

$z u$ 祖

$z i$ 子 
